\title{
BMJ Open Group-based exercise in daily clinical practice to improve physical fitness in men with prostate cancer undergoing androgen deprivation therapy: study protocol
}

\author{
Peter Østergren, ${ }^{1,2}$ Anne-Mette Ragle, ${ }^{3}$ Henrik Jakobsen, ${ }^{1}$ \\ Tobias Wirenfeldt Klausen, ${ }^{4}$ Anders Vinther, ${ }^{3}$ Jens Sønksen ${ }^{1,2}$
}

To cite: Østergren $P$,

Ragle A-M, Jakobsen $\mathrm{H}$, et al. Group-based exercise in daily clinical practice to improve physical fitness in men with prostate cancer undergoing androgen deprivation therapy: study protocol. BMJ Open 2016;6: 011460.

doi:10.1136/bmjopen-2016011460

- Prepublication history for this paper is available online. To view these files please visit the journal online (http://dx.doi.org/10.1136/ bmjopen-2016-011460).

Received 9 February 2016 Revised 2 May 2016 Accepted 26 May 2016

CrossMark

For numbered affiliations see end of article.

Correspondence to Dr Peter Østergren; peter. busch.oestergren@regionh.dk

\section{ABSTRACT}

Introduction: Level 1 evidence supports the use of supervised exercise to mitigate the adverse effects of androgen deprivation therapy (ADT) in men with prostate cancer. The data, however, have been generated in controlled research settings and might not be transferable to daily clinical practice. This article describes the design of an ongoing prospective observational study to evaluate the potential benefits of exercise in daily clinical practice.

Methods and analysis: Men diagnosed with prostate cancer starting or already receiving ADT at our facility are invited to participate in a 12-week exercise programme implemented as the standard of care. Exclusion criteria are opioid-demanding treatment for skeletal pain, an Eastern Cooperative Oncology Group (ECOG) performance status above 2 or the inability to perform floor and machine exercises independently. The intervention consists of an initial educational session of $1 \frac{1}{2}$ hours followed by 12 weeks of group-based supervised training two times a week. The focus of the exercise is progressive resistance training in combination with aerobic training. Participants are measured at baseline, after 12 weeks and after 24 weeks as part of the programme. Primary endpoints of this study are changes in physical fitness evaluated by the $30 \mathrm{~s}$ Chair-Stand Test and Graded Cycling Test with Talk Test. Secondary endpoints include changes in quality of life, body composition and safety of exercise. Inclusion started in August 2014, with 169 participants being included by December 2015.

Ethics and dissemination: The study has been reviewed by the Scientific Ethical Committee of the Capital Region of Denmark (reference number H-3-2013-FSP39). The results of the study will be published in peer-reviewed international journals and will be presented at national and international conferences and symposiums.

Trial registration number: NCT02631681; Pre-results.

\section{INTRODUCTION}

Androgen deprivation therapy (ADT) is used in the treatment of prostate cancer to

\section{Strengths and limitations of this study}

- This trial will include a large sample size and reports on the potential benefits of an exercise intervention programme implemented in daily clinical practice.

- This is the first larger trial to specifically report on safety of resistance training for men with prostate cancer and bone metastases.

- The trial includes a structured transition to out-of-hospital training and a 12-week postintervention follow-up, thereby addressing the potential difficulties of maintaining the positive effects of exercise.

- The study lacks randomisation.

improve survival, delay disease progression and to reduce cancer-related morbidity. ${ }^{1}$ At the same time, the castrate levels of testosterone reached by ADT is associated with several side effects, including loss of physical fitness, decreased quality of life (QoL) and metabolic impairments, potentially leading to treatment-induced morbidity. ${ }^{2-5}$ Often, patients report increased fatigue and loss of strength, ${ }^{6}{ }^{7}$ supported by studies showing ADT to decrease lean body mass as well as muscle strength. ${ }^{8}$ Increased fat mass, decreased insulin sensitivity and impaired glucose metabolism are also well-known side effects of ADT. ${ }^{9}{ }^{10}$ It is therefore not surprising that population-based cohort studies have reported an increased risk of bone fracture, diabetes and possibly cardiovascular disease (CVD) for men undergoing ADT compared with non-ADT users. ${ }^{3-5} 11$

Exercise is recommended as a mitigating treatment to the aforementioned adverse effects, but results supporting the use of exercise have been primarily generated in 
controlled research settings and may not be transferable to daily clinical practice. A recent systematic review and meta-analysis investigated the effect of exercise in men with prostate cancer and found exercise to improve cancer-specific fatigue, QoL and physical fitness and function. ${ }^{12}$ The evidence was primarily obtained from men with prostate cancer receiving ADT. This conclusion is similar to those made in previous systematic reviews exploring the effect of exercise in men with prostate cancer undergoing ADT. ${ }^{1314}$ The strongest data currently come from randomised trials where the intervention arm has included supervised group-based exercise combining resistance and aerobic training two times a week for at least 12 weeks. ${ }^{15-17}$ A characteristic of these positive trials has been adherence rates to the intervention above $80 \%$ with interventions being performed in a controlled research setting. ${ }^{15-17}$ Of note is the trial by Bourke et al, ${ }^{16}$ in which they randomised 100 men undergoing long-term ADT to supervised combined aerobic and resistance exercise or usual care. Follow-ups were at 12 weeks with participants ending intervention and additionally at 6 months. The researchers reported an adherence rate of $94 \%$ for the supervised exercise session, but after the withdrawal of supervision, only $68 \%$ attended the follow-up at 6 months. In addition, they found a positive effect of exercise on QoL measured postintervention, but the beneficial changes were not sustained at 6 months, highlighting the difficulty of maintaining continued exercise and the benefits thereof outside the controlled research setting. This yields two unanswered questions if a similar exercise programme were offered in a daily clinical setting: (1) will similar positive effects on physical fitness, body composition and QoL be reachable? (2) How can continued exercise after a 12-week intervention be supported?

Another concern is that only a few previous studies have included participants with bone metastases. ${ }^{16}$ 18-20 Cormie $e t a l^{20}$ performed a safety study including only 20 participants with bone metastases randomised to resistance training or usual care and found resistance training to be safe and well tolerated. However, exercises targeting affected regions with bone metastases were avoided. As many patients with disseminated prostate cancer have or will develop multiple regions affected by metastatic bone disease, the safety profile of the study by Cormie et al cannot be generalised to a broader group of men with metastatic prostate cancer.

Hence, the aims of this study are as follows:

1. Examine the potential benefits of exercise implemented in daily clinical practice on physical fitness (primary aim), QoL and body composition in men with prostate cancer undergoing ADT.

2. Examine whether a structured transition to outof-hospital training can maintain the effects obtained during supervised exercise intervention.

3. Assess the safety of progressive resistance training in men with metastatic prostate cancer.
Our hypotheses are as follows: (1) a patient educational session followed by a 12-week, supervised, group-based progressive resistance training programme implemented as the standard of care in a normal clinical setting will improve physical fitness, body composition and QoL. (2) A structured transition to out-of-hospital training will maintain the improvements in physical fitness, body composition and patient QoL after the exercise intervention. (3) Progressive resistance training using machines is also a safe and feasible method of training for men with prostate cancer and bone metastases.

This study will contribute to the current knowledge regarding the benefits of supervised exercise when implemented in daily clinical practice and regarding the potential difficulties when transitioning to out-of-hospital training. Furthermore, a safety assessment of resistance training for men with metastatic prostate cancer will be made, which is critically needed for exercise to be generally recommended to men undergoing ADT.

\section{METHODS AND ANALYSIS}

This is a prospective observational study assessing an exercise programme implemented as a standard of care and routinely offered to men with prostate cancer receiving ADT at our facility. A schematic overview of the programme is depicted in figure 1. Participants are recruited from a single centre and participate in a patient educational programme of $1 \frac{1}{2}$ hours followed by 12 weeks of combined aerobic and resistance group-based training. Participants will be evaluated at baseline, after the exercise intervention and 12 weeks postintervention.

\section{Participants}

Men with prostate cancer undergoing ADT (orchiectomy, luteinising hormone-releasing hormone agonists or antagonists) are invited to participate in the exercise programme when attending their ambulant prostate cancer controls at the Department of Urology, Herlev and Gentofte University Hospital, Denmark. ADT must be continued for at least 1 year forward. Thus, the cohort consists of patients receiving ADT as an adjuvant treatment in combination with external beam radiation with curative intent and patients with advanced metastatic prostate cancer. Patients undergoing chemotherapy or second-line hormonal therapy with enzalutamide or abiraterone in addition to ADT are also eligible for participation. Exclusion criteria are opioid-demanding treatment for skeletal pain, Eastern Cooperative Oncology Group (ECOG) performance status above 2 or the inability to perform floor and machine exercises independently. Patients with osteoporosis or a larger bone metastatic burden assessed by the referring physician undergo a modified resistance training programme. Prospective data collection began in August 2014 and is expected to continue until April 2017. The number of participants, who have given consent to the use of their healthcare data, was 169 in December 2015. 
Figure 1 Schematic overview of the patient programme (blue blocks) and the test visits (green blocks).

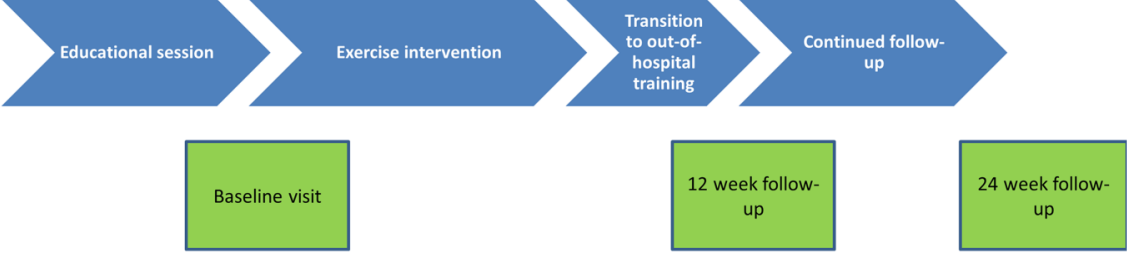

\section{Intervention}

The programme is a standard of care (implemented at our facility in December 2013) being offered to all our patients receiving ADT who meet the criteria for participation. The intervention consists of an educational session followed by 12 weeks of supervised group-based exercise, ending with a structured out-of-hospital transition to continued exercise at a local training facility.

The educational session lasts $1 \frac{1 / 2}{2}$ hours, with lectures from a urologist, nurse, dietitian and a physiotherapist specially assigned to this task. Patients are invited to bring a person of confidence and to attend with the group they are going to start the training programme with. During the educational session, the natural history of prostate cancer is covered as well as the rationale, indications and methods of action for ADT. Furthermore, known physical and psychological side effects of ADT, including the implications of decreased libido and risks of osteoporosis, diabetes and potential CVD, are addressed. The dietitian lectures on the latest dietary recommendations according to the Danish Health Authorities.

The following exercise programme consists of $1 \frac{1}{2}$ hours of supervised group-based training two times a week for 12 weeks combining aerobic and resistance training. This exercise routine has shown positive effects on physical fitness, body composition and QoL in several randomised clinical trials. ${ }^{15-17} 1921$ The main focus of this programme is progressive resistance training using machines, following the guidelines for progression recommended by the American College of Sports Medicine (ACSM) ${ }^{22}$ Each session is started with $15 \mathrm{~min}$ of warm-up on a bike or stair walking followed by $60 \mathrm{~min}$ of core, upper and lower body resistance training using machines. The programme consists of seven exercises targeting the major muscle groups of the body-leg press, knee extensions, hamstring curls, chest press, low row, sit-ups and back extension exercises. The progression model outlined in table 1 is used as a general guideline to the individualised training progression. The resistance training programme is supplemented by aerobic exercise- $\sim 6$ min of exercise on a treadmill and $500 \mathrm{~m}$ of ergometer rowing. The resistance training is tailored to the individual participant by the physiotherapist to accommodate both his strength level and potential hazards such as osteoporosis and major regions of metastatic disease. Close attention is paid if the physician has highlighted training restrictions in the patient file due to comorbidity. In the presence of multiple bone metastases or osteoporosis, a symptom-dependent and very conservative approach to the progression of the resistance training programme is adopted-that is, very small increments in resistance. In addition to the supervised exercise, participants are encouraged to perform one exercise session at home each week according to a given programme provided in the patient educational material; furthermore, both the participants and, if possible, their partners are instructed to stay generally physical active, defined as daily non-strenuous activity of 10000 paces.

The main behaviour change techniques according to the the 'Coventry, Aberdeen, and London-Refined' (CALO-RE) taxonomy ${ }^{23}{ }^{24}$ used in this programme are: 'provide information on consequences of behaviour in general', 'goal setting (behaviour)', 'prompt practice', 'prompting generalisation of a target behaviour', 'provide instruction on how to perform the behaviour', 'model/demonstrate the behaviour' and 'plan social support/social change'. The supervised group-based training allows for verbal and visual instruction on how to exercise and creates a platform where social support and bonds between the participants are created. Furthermore, the last two supervised training sessions are performed at a local commercial fitness centre to familiarise participants with available facilities in the community and to make a structured transition to out-of-hospital training. Participants are encouraged to stay in contact with one another and to continue the group-based training. For motivational purposes and to monitor the effect on physical fitness, the participants are tested at baseline and after 12 and 24 weeks. Concurrently, the participants are seen by a nurse to monitor changes in body composition as well as to address any concerns or questions the participant might have regarding both his cancer treatment and the exercise programme.

\section{Measurements}

All measurements of primary and secondary outcomes are validated and widely used in clinical research. All participants will be assessed at baseline, postintervention and at the 24-week follow-up by specially trained nurses and physiotherapists. A loss to follow-up of $\sim 10 \%$ at 24 weeks is expected primarily due to symptomatic disease progression. Handling of missing data is covered under sample size and statistical analysis.

\section{Primary study endpoints}

The primary endpoints of this study are a change in workload (Watt) during the Graded Cycling Test with Talk Test (GCT-TT) and a change in the number of repetitions using the $30 \mathrm{~s}$ Chair-Stand Test (30s-CST). 
Table 1 Progression model for the resistance training over 24 sessions

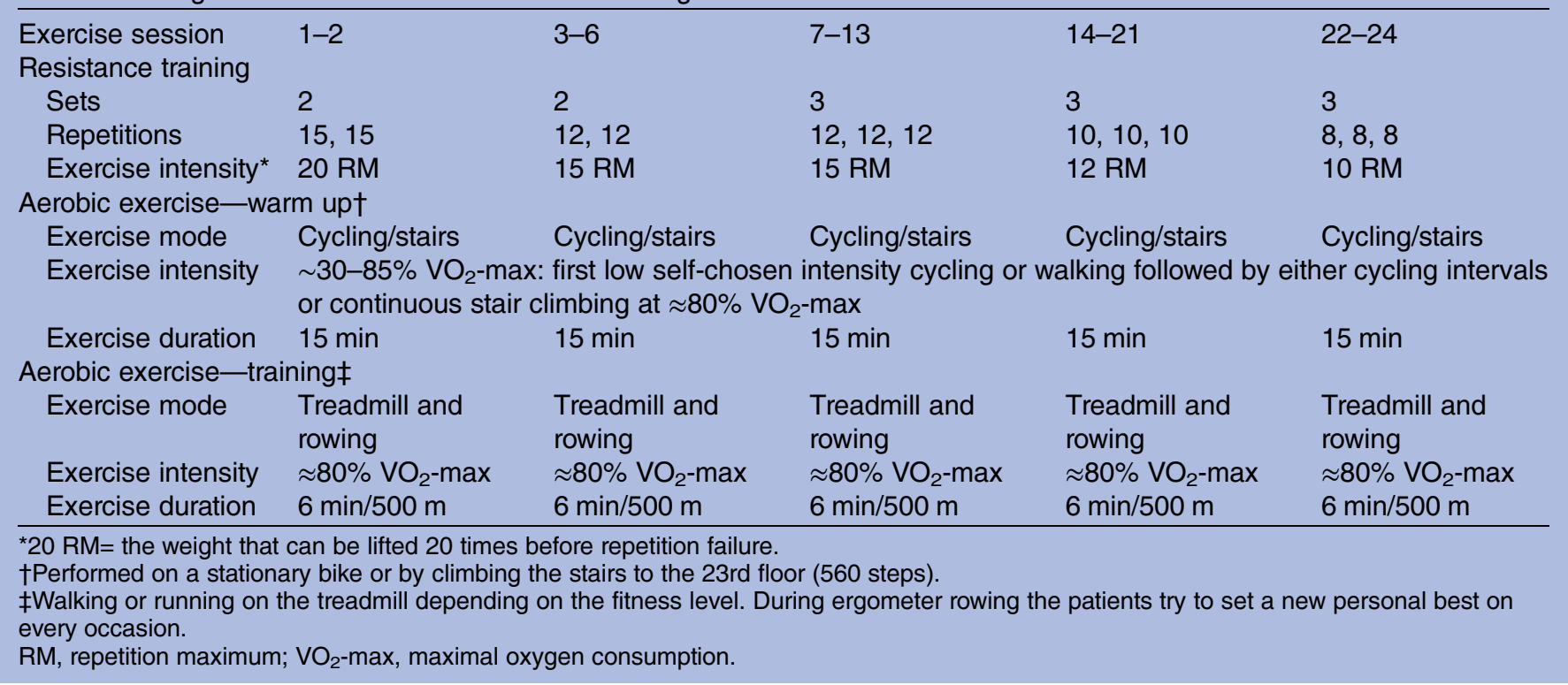

Both endpoints are measured as the within-person change from baseline. GCT-TT is a submaximal exercise test used to assess aerobic fitness. It is not dependent on the measurement of heart rate and is thus not influenced by medications or comorbidities potentially affecting the heart rate variability. Excellent test/retest reliability as well as an acceptable measurement error and good responsiveness have been observed in cardiac patients. ${ }^{25} 26$ Although not directly validated in patients with prostate cancer, the GCT-TT is expected to be feasible and reliable in this population because the patients are expected to resemble the populations of cardiac patients regarding age and comorbidity. The GCT-TT is performed on a cycle ergometer as described by Nielsen et $a l^{25}$ A constant pedalling rate of 60 rounds per minute is maintained throughout the test. After an initial 2-min warm-up period with the exercise intensity set to $15 \mathrm{~W}$, a ramp protocol is applied with $15 \mathrm{~W}$ increases every minute. During the last $10 \mathrm{~s}$ of each exercise step of the test, a standard, 30-word text passage is recited by the patients. Subsequently, they are asked, 'Are you still able to speak comfortably?'. The following predefined answers can be given: 'yes', 'unsure' or 'no', corresponding to a positive talk test ( $\mathrm{TT}+)$, equivocal talk test $(\mathrm{TT} \pm)$ and negative talk test $(\mathrm{TT}-)$. When the answer 'no' is given, the test ends and the exercise intensity is recorded.

The 30s-CST is used to assess functional performance/lower extremity muscle strength and endurance. ${ }^{27}$ The 30s-CST was chosen because it has previously been used in patients with cancer undergoing a similar exercise intervention mainly consisting of progressive resistance training. ${ }^{28}$ The improvement in the 30s-CST result $(\approx 15-16 \%)$ corresponded well with the improvement in isokinetic knee extension strength $(\approx 12-18 \%)$ measured by an isokinetic dynamometer. ${ }^{28}$ The $30 \mathrm{~s}$-CST is performed according to the test manual described by Jones et $a l,{ }^{27}$ as follows: a chair with a seat height of $43 \mathrm{~cm}$ is used. With arms crossed over the chest as many rises as possible is performed in $30 \mathrm{~s}$. The starting position is sitting on the chair. A complete rise is recorded when the knees and hips are fully extended in a standing position and the next rise can begin when the patient has assumed a sitting position on the chair seat without touching the back rest. The command 'ready, set, go' is used. The clock is started on 'go', and the number of complete rises in $30 \mathrm{~s}$ is counted. The modified version of the 30s-CST, in which the patients actively use the armrests, is used in patients who are unable to stand up only using the legs. ${ }^{29}$

\section{Secondary study endpoints}

Questionnaire-based assessments are conducted using the validated European Organisation for Research and Treatment of Cancer Quality of Life Core Questionnaire (EORTC QLQC-30 V.3.0) and Quality of Life Prostatespecific Questionnaire (EORTC QLQ-PR25). ${ }^{30} 31$ Specific endpoints of the EORTC QLQC-30 questionnaire are changed from baseline in global health status, functional scales (physical functioning, role functioning, emotional functioning, cognitive functioning and social functioning) and fatigue measured by the symptom scale. The EORTC QLQ-PR25 questionnaire is used to measure changes from baseline in sexual activity and sexual functioning. The latter will only be analysed for patients who are sexually active at baseline. Furthermore, the change in ADT-related symptoms is assessed by the hormonal treatment-related symptoms scale of the EORTC QLQ-PR25. 
To examine changes in body composition, nurses measure the body mass index $\left(\mathrm{BMI}, \mathrm{kg} / \mathrm{m}^{2}\right.$ ) and the waist circumference (WC) and hip circumference (HC) in centimeters. A secondary end point is the change from baseline in BMI, WC, HC and waist-hip ratio. These changes do not reflect the benefits of exercise on muscle mass. However, waist-hip ratio and WC are used to assess change in fat distribution and abdominal adipose tissue, which are risk factors of the metabolic syndrome, diabetes mellitus and CVD. ${ }^{32} \mathrm{WC}$ is measured according to the WHO guidelines midway between the inferior margin of the last rib and the crest of the ilium, in the horizontal plane. ${ }^{33}$ Similarly, HC is measured around the buttocks in the horizontal plane at the level of maximum extension.

Blood pressure (systolic and diastolic) is measured at each visit after a 10 min seated rest. Changes in systolic and diastolic blood pressure from baseline will be investigated separately.

Safety of exercise is measured by the prospective recording of any serious adverse events in relation to training. A serious related adverse event is defined in accordance with the European Medicine Agency (EMA) as any undesirable experience associated with exercise that results in death, is life-threatening, requires hospitalisation or prolongation of existing hospitalisation, results in persistent or significant disability or results in some other medically important condition. Minor adverse events such as muscle strains or muscle soreness after exercise will not be reported.

Compliance with the programme will be reported as attendance at the supervised group training sessions. The ability of participants to perform the exercises as prescribed, that is correct intensity and number of repetitions, will be reported as percentage of exercises during attended sessions completed as prescribed. Patient self-reports, completed during each session, on intensity and the number of repetitions done for each exercise will be used for this analysis. Furthermore, continued exercise after the ended intervention will be reported as either yes or no and also relies on patient self-reports.

\section{Sample size and statistical analysis}

An inclusion cut-off date has been set to 15 October 2016 with expectations of having included $\sim 230$ participants. Previous larger randomised trials investigating the effects of exercise in men with prostate cancer have included $\sim 100$ participants. ${ }^{162134}$ As we mean to evaluate the safety of exercise and there is no control arm, we have chosen a substantially larger sample size. Minimal sample size calculations to detect a clinically significant change have been carried out for the primary endpoints. Data from an in-house project have shown clinical significance within-person change in the GCT-TT at $13 \mathrm{~W}$ and the SD of the change at $18 \mathrm{~W}^{26}$ As we expect to perform multiple tests, the $\alpha$-level in the sample size calculation is set at 0.005 , two-sided, to allow correction for multiple testing. With a power at 0.80 , a minimal sample size of 30 participants is required. For the 30s-CST, the clinically significant change was set at 2.6 repetitions with an SD at 2.2 in accordance with a previous study by Wright et $a l^{35}$ With a power at 0.80 and a significance level at 0.005 , a sample size of 13 is necessary.

Linear mixed-effect models or Friedman tests will be used for analyses of data with multiple time points. Clinically relevant covariates such as age, exercise compliance (high compliance is predefined as $75 \%$ attendance), continued exercise postintervention, duration of ADT, baseline BMI, treatment for castration-resistant prostate cancer other than ADT and known comorbidities (CVD, diabetes mellitus, hypertension, dyslipidaemia and osteoporosis) will be included in the analyses to assess effect modification (interaction). If a significant interaction is found we will perform subgroup analysis on the covariate. Missing data will be handled by the mixed-effect models, assuming the data are missing at random. A sensitivity analysis for the missing-at-random assumption will be performed. Secondary, and more explorative, analyses will include comparisons of the baseline to the 12-week follow-up and of the 12-week to the 24-week follow-up.

$\mathrm{p}$ Values will be two-sided and $\mathrm{p}$ values below 0.05 will be considered significant. Statistical calculations will be performed using SAS 9.4 (SAS Institute, Cary, North Carolina, USA) or a similar software. Statistical planning was conducted in cooperation with a biostatistician.

\section{ETHICS AND DISSEMINATION}

The ethical committee did not find approval to be required. All participants have provided written informed consent. The study has been registered on Clinicaltrials.gov (NCT02631681). Results of the study will be published in peer-reviewed international journals or otherwise made publicly available and will be presented at national and international conferences and symposiums irrespective of the outcomes. Completion of this study is expected by spring 2017, and dissemination of the results will begin as soon as possible thereafter.

\section{DISCUSSION}

The aim of this study is to report results on exercise benefits in a supervised setting and when participants transition from supervised to individual training for men with prostate cancer receiving ADT. The strength of this study is that the intervention is part of the daily clinical practice as a standard of care in our department. Therefore, the participants are also comparable to those seen by the treating physicians. The present study with a large sample size will also answer the important question of whether resistance training is safe for men with bone metastases, thereby making recommendations on exercise programmes for men undergoing ADT, as a whole, easier. The most important limitation of the study is that 
we do not have a control group as a reference because we are reporting data on an intervention implemented as the standard of care. As we are examining the withinperson change over time, we expect the latter to be a potential influential factor. Participants with metastatic prostate cancer are with the 24-week follow-up prone to get worse from their disease and potentially develop further ADT-related side effects. Thus, time-related worsening can in itself hide a positive effect of the exercise intervention. However, this will only strengthen a potential significant positive result. Successful outcomes of this study will support the implementation of exercise programmes for men with prostate cancer receiving ADT in clinical practice.

\section{Author affiliations}

${ }^{1}$ Department of Urology, Herlev and Gentofte University Hospital, Herlev, Denmark

${ }^{2}$ Faculty of Health and Medical Sciences, University of Copenhagen,

Copenhagen, Denmark

${ }^{3}$ Department of Rehabilitation, Herlev and Gentofte University Hospital, Herlev, Denmark

${ }^{4}$ Department of Haematology, Herlev and Gentofte University Hospital, Herlev, Denmark

Contributors All the authors contributed to the study conception and design. $P \emptyset$ and $\mathrm{HJ}$ contributed to obtaining funding. $\mathrm{P} \emptyset, \mathrm{AV}$ and $\mathrm{A}-\mathrm{MR}$ drafted the manuscript. Biostatistician TWK planned the statistical analyses in cooperation with $\mathrm{P} \varnothing$ and $\mathrm{AV}$. All the authors reviewed the manuscript and provided comments and revisions; and read and approved the final manuscript.

Funding This study is supported by a research donation by Ferring Pharmaceuticals A/S. Ferring Pharmaceutical A/S also provided a booklet on exercise and health for men with prostate cancer on androgen deprivation therapy given to the participants based on the FEEL+ concept.

Disclaimer Ferring Pharmaceuticals A/S has no access or rights to the data generated in this study and have not contributed to the concept, design or submission process.

\section{Competing interests None declared.}

Ethics approval The study has been reviewed by the Scientific Ethical Committee of the Capital Region of Denmark (reference number H-3-2013-FSP39).

Provenance and peer review Not commissioned; externally peer reviewed.

Open Access This is an Open Access article distributed in accordance with the Creative Commons Attribution Non Commercial (CC BY-NC 4.0) license, which permits others to distribute, remix, adapt, build upon this work noncommercially, and license their derivative works on different terms, provided the original work is properly cited and the use is non-commercial. See: http:// creativecommons.org/licenses/by-nc/4.0/

\section{REFERENCES}

1. Pagliarulo V, Bracarda S, Eisenberger MA, et al. Contemporary role of androgen deprivation therapy for prostate cancer. Eur Urol 2012;61:11-25.

2. Hussain M, Tangen CM, Berry DL, et al. Intermittent versus continuous androgen deprivation in prostate cancer. $N$ Engl $\mathrm{J} \mathrm{Med}$ 2013;368:1314-25.

3. O'Farrell S, Garmo H, Holmberg L, et al. Risk and timing of cardiovascular disease after androgen-deprivation therapy in men with prostate cancer. J Clin Oncol 2015;33:1243-51.

4. Keating NL, O'Malley AJ, Smith MR. Diabetes and cardiovascular disease during androgen deprivation therapy for prostate cancer. $J$ Clin Oncol 2006;24:4448-56.
5. Shahinian VB, Kuo YF, Freeman JL, et al. Risk of fracture after androgen deprivation for prostate cancer. N Engl J Med 2005;352:154-64.

6. Stone $\mathrm{P}$, Hardy J, Huddart R, et al. Fatigue in patients with prostate cancer receiving hormone therapy. Eur J Cancer 2000;36:1134-41.

7. Herr HW, O'Sullivan M. Quality of life of asymptomatic men with nonmetastatic prostate cancer on androgen deprivation therapy. $J$ Urol 2000;163:1743-6.

8. Finkelstein JS, Lee H, Burnett-Bowie SA, et al. Gonadal steroids and body composition, strength, and sexual function in men. $N$ Engl J Med 2013;369:1011-22.

9. Smith MR, Lee H, Fallon MA, et al. Adipocytokines, obesity, and insulin resistance during combined androgen blockade for prostate cancer. Urology 2008;71:318-22.

10. Smith MR, Lee $\mathrm{H}$, Nathan DM. Insulin sensitivity during combined androgen blockade for prostate cancer. J Clin Endocrinol Metab 2006;91:1305-8.

11. Jespersen CG, Nørgaard M, Borre M. Androgen-deprivation therapy in treatment of prostate cancer and risk of myocardial infarction and stroke: a nationwide Danish population-based cohort study. Eur Urol 2014:65:704-9.

12. Bourke L, Smith $D$, Steed L, et al. Exercise for men with prostate cancer: a systematic review and meta-analysis. Eur Urol 2016;69:693-703.

13. Gardner JR, Livingston PM, Fraser SF. Effects of exercise on treatment-related adverse effects for patients with prostate cancer receiving androgen-deprivation therapy: a systematic review. $J$ Clin Oncol 2014;32:335-46.

14. Hasenoehrl T, Keilani M, Sedghi Komanadj T, et al. The effects of resistance exercise on physical performance and health-related quality of life in prostate cancer patients: a systematic review. Support Care Cancer 2015;23:2479-97.

15. Galvão DA, Taaffe DR, Spry N, et al. Combined resistance and aerobic exercise program reverses muscle loss in men undergoing androgen suppression therapy for prostate cancer without bone metastases: a randomized controlled trial. $J$ Clin Oncol 2010;28:340-7.

16. Bourke L, Gilbert S, Hooper R, et al. Lifestyle changes for improving disease-specific quality of life in sedentary men on long-term androgen-deprivation therapy for advanced prostate cancer: a randomised controlled trial. Eur Urol 2014;65:865-72.

17. Cormie P, Galvão DA, Spry N, et al. Can supervised exercise prevent treatment toxicity in patients with prostate cancer initiating androgen-deprivation therapy: a randomised controlled trial. BJU Int 2015;115:256-66

18. Winters-Stone KM, Dobek JC, Bennett JA, et al. Skeletal response to resistance and impact training in prostate cancer survivors. Med Sci Sport Exerc 2014:46:1482-8.

19. Bourke L, Doll H, Crank $\mathrm{H}$, et al. Lifestyle intervention in men with advanced prostate cancer receiving androgen suppression therapy: a feasibility study. Cancer Epidemiol Biomarkers Prev 2011;20:647-57.

20. Cormie P, Newton RU, Spry N, et al. Safety and efficacy of resistance exercise in prostate cancer patients with bone metastases. Prostate Cancer Prostatic Dis 2013;16: 328-35.

21. Galvão DA, Spry N, Denham J, et al. A multicentre year-long randomised controlled trial of exercise training targeting physical functioning in men with prostate cancer previously treated with androgen suppression and radiation from TROG 03.04 RADAR. Eur Urol 2014:65:856-64.

22. American College of Sports Medicine. American College of Sports Medicine position stand. Progression models in resistance training for healthy adults. Med Sci Sports Exerc 2009;41:687-708.

23. Michie S, Ashford S, Sniehotta FF, et al. A refined taxonomy of behaviour change techniques to help people change their physical activity and healthy eating behaviours: the CALO-RE taxonomy. Psychol Health 2011;26:1479-98.

24. Bourke L, Homer KE, Thaha MA, et al. Interventions for promoting habitual exercise in people living with and beyond cancer. Cochrane Database Syst Rev 2013;9:CD010192.

25. Nielsen SG, Buus L, Hage T, et al. The Graded Cycling Test combined with The Talk Test is reliable for patients with ischemic heart disease. J Cardiopulm Rehabil Prev 2014;34: 276-80.

26. Nielsen SG, Vinther A. Graded Cycling Test combined with Talk Test is responsive in cardiac rehabilitation. $J$ Cardiopulm Rehabil Prev Published Online First: 29 Apr 2016. doi:10.1097/HCR. 000000000000180 
27. Jones CJ, Rikli RE, Beam WC. A 30-s Chair-stand Test as a measure of lower body strength in community-residing older adults. Res Q Exerc Sport 1999;70:113-19.

28. Lønbro S, Dalgas U, Primdahl H, et al. Feasibility and efficacy of progressive resistance training and dietary supplements in radiotherapy treated head and neck cancer patients-the DAHANCA 25A study. Acta Oncol (Madr) 2013;52:310-18.

29. Rikli RE, Jones CJ. Senior Fitness Test Manual. Champaign, IL: Human Kinetics, 2001.

30. Aaronson NK, Ahmedzai S, Bergman B, et al. The European Organization for Research and Treatment of Cancer QLQ-C30: a quality-of-life instrument for use in international clinical trials in oncology. J Natl Cancer Inst 1993;85:365-76.

31. van Andel G, Bottomley A, Fosså SD, et al. An international field study of the EORTC QLQ-PR25: a questionnaire for assessing the health-related quality of life of patients with prostate cancer. Eur $J$ Cancer 2008;44:2418-24.
32. Alberti KGMM, Eckel RH, Grundy SM, et al. Harmonising the metabolic syndrome: a joint interim statement of the International Diabetes Federation Task Force on Epidemiology and Prevention; National Heart, Lung, and Blood Institute; American Heart Association; World Heart Federation; International Association for the Study of Obesity. Circulation 2009;120:1640-5.

33. Physical status: the use and interpretation of anthropometry. Report of a WHO Expert Committee. World Health Organ Tech Rep Ser 1995;854:1-452.

34. Segal RJ, Reid RD, Courneya KS, et al. Resistance exercise in men receiving androgen deprivation therapy for prostate cancer. $J$ Clin Oncol 2003:21:1653-9.

35. Wright AA, Cook CE, Baxter GD, et al. A comparison of 3 methodological approaches to defining major clinically important improvement of 4 performance measures in patients with hip osteoarthritis. J Orthop Sport Phys Ther 2011;41:319-27. 